\title{
Geometrical Model Based Refinements in Nanotube Chiral Indices
}

\author{
Levan Chkhartishvili, Tamar Berberashvili \\ Department of Physics, Georgian Technical University, Tbilisi, Georgia \\ E-mail: chkharti2003@yahoo.com \\ Received March 28, 2011; revised April 6, 2011; accepted April 13, 2011
}

\begin{abstract}
There is demonstrated how it is possible to refine graphitic nanotubes' chiral indices based on the appropriate geometrical model for their structure.
\end{abstract}

Keywords: Graphitic Nanotube, Diameter, Chiral Indices, Analytical Polyhedral Model

\section{Introduction}

Nowadays there known many types of nanostructures. But certainly graphitic carbon $\mathrm{C}$ nanotubes discovered by Iijima [1] are the most prominent member of a nanomaterials family with technologically interesting properties. Many researchers have been successful in producing single- and multi-walled carbon nanotubes with various diameters and lengths showing that they exhibit number of new properties and have the potential to be very useful in the design of nanomechanical and nanoelectronic devices. Nowadays carbon fibers are used as conductive fillers and mechanical structural reinforcements in composites, e.g., in the automobile and aerospace industry, materials for sporting-goods production, scanning probe tips, electrode additive materials exploiting their resiliency, e.g., in lithium ion battery applications, electrostatic shielding materials, specialized medical appliances, like the catheters, etc. Nanotubular carbon has revealed a potential to be used also as supercapacitor electrodes, single-tip electron guns, multi-tip array X-ray sources, 3D-electrostatic dampers, probe array test systems, fuel-cell electrodes and catalyst supports, brush contacts, transparent conducting films, sensor devices and biosensors, field emission displays/lightings, electromechanical memory devices, inks for printing, thermal-management systems, power transmission cables, nanoelectronic and flexible electronic devices, photovoltaic devices, filtration/separation membranes, drug-delivery systems, etc. The most recent survey on carbon nanotubes' applications one can find in [2].

Based upon the similarity of structures of the boron nitride BN layered phases with graphite, it was assumed that along with carbon nanotubes, stable $\mathrm{BN}$ nanotubes - fragments of hexagonal BN layers wrapped into cylinders - could also exist [3]. Soon after synthesizing the first BN nanotubes, it was proposed a number of their possible applications in technique, like the fibers, composites, nanotubular heterojunctions, nanoreservoirs, piezoelectrics, hypersound quantum generators, etc (e.g., see [4-13]). Interestingly that there are also some other binary compounds not crystallizing in layered graphitelike bulk structures, but nevertheless forming hexagonal nanotubes: AlN [14], GaN [15], ZnO [16-19], and $\mathrm{ZnS}$ $[20,21]$.

Carbon nanotubes and other (binary compounds) graphitic ones are a heterogeneous group of materials. Such nanotubular material can comprise structurally perfect single-walled nanotubes with a quite wide range chiral indices, multi-walled nanotubes, nanotube bundles, and single-walled nanotubes those change chirality along their length due to structural defects. Furthermore, they can interact with the environment and other neighboring nanosystems. These two are main reasons why various spectroscopic techniques used to characterize individual nanotubes usually require long data acquisition times and complicated sample preparation. New insights into the structure-lines in graphitic single-wall nanotubes can provide a combination of experimental data with theoretical calculations based on the geometrical models for their atomic structure.

Present work aims to illustrate how an appropriate geometrical model can be used in structural data refinement recently obtained for single-walled carbon nanotubes. Namely, indices will be specified for nanotubes with given diameters. Paper is organized as follows. Af- 
ter Introduction the brief overview is given on geometrical models up to this point suggested for graphitic single-walled nanotubes. Then, one of them-analytical polyhedral model - is applied to the recent experimental data analysis. And finally, some concluding remarks are given.

\section{Geometrical Models for Graphitic Nanotubular Structures}

\subsection{Conventional Rolled-up Model}

In all but a few of studies dealing with carbon nanotubes curvature effects are ignored and such structures are assumed to be constructed by rolling up a plane sheet of graphene, which in its plane state is assumed to comprise a network of perfect hexagons, in the sense that all bond lengths and all bond angles are assumed to be identical. The conventional rolled-up model [22-24] implies that the nanotube diameter $D_{(n, m)}$ is assumed to be given by the expression

$$
\frac{D_{(n, m)}}{d_{(n, m)}}=\frac{\sqrt{3\left(n^{2}+n m+m^{2}\right)}}{\pi}
$$

where $d_{(n, m)}$ denotes the bond length, and $n=1,2,3, \cdots$ and $0 \leq m \leq n$ are nanotube indices.

Mentioned model with same success can be applied to nanotubes of other materials with graphitic structure, like the boron nitride $\mathrm{BN}$ nanotubes [25].

\subsection{Idealized Polyhedral Model}

But, in the cylindrical rolled-up state, the bond lengths and bond angles are no longer equal and if such discrepancies are not small they should not be ignored in regard to the calculation for the diameter of the carbon nanotube. Taking into account these circumstances, Cox and Hill have constructed a new geometrical model for carbon nanotubes [26-28] called by authors as idealized polyhedral model. It adopts as its basic hypothesis that the hexagons in the cylindrical rolled-up state should be ideally perfect in the sense that all bond lengths and all bond angles are identical and furthermore that all atoms are equidistant from a common axis of symmetry. These three assumptions give rise to a geometric structure for which all bonds play a truly equal role, unlike conventional theory. It has been demonstrated that the idealized polyhedral model gives rise to the conventional expression of the diameter as the leading term, but in addition one may include correction terms. In general, the smaller the tube diameter, the larger these correction terms become.
In order to find the nanotube diameter expression in the idealized polyhedral model, one need to introduce a further two angles which are termed as the subtend semi-angle $\psi$ and the incline angle $\omega$, and a further three parameters $\lambda, \mu$ and $v$, which are defined in terms of the various angles. The first step in determining the exact geometric structure is to determine the subtend angle $2 \psi$, subtended at the axis of the cylinder by the base of a single equilateral triangle. This angle is determined as a root of the following transcendental equation

$$
\begin{aligned}
& \left(n^{2}-m^{2}\right) \sin ^{2}((n+m) \psi-\pi) / m \\
& -n(n+2 m) \sin ^{2}(n \psi-\pi) / m+m(2 n+m) \sin ^{2} \psi=0 .
\end{aligned}
$$

This equation may have many roots but specifically the one required here must also satisfy the inequalities

$$
n \psi \leq \pi \leq(n+m) \psi .
$$

Once the correct root is found all other expressions can be determined. The chiral angle $\theta$ is given by the expression

$$
\cos ^{2} \theta=\frac{n(n+2 m) \sin ^{2} \psi}{(n+m)^{2} \sin ^{2} \psi-m^{2} \sin ^{2}((n+m) \psi-\pi) / m}
$$

and the angle of incline $\omega$ of the pyramidal components of the surfaces is given by

$$
\sin \omega=\frac{\sqrt{\cot ^{2} \psi+4 \cos ^{2} \theta-3}-\cot \psi}{\sqrt{3} \cos \theta} .
$$

With all the necessary angles now determined the bond angle $2 \phi$ is determined from the formula

$$
\sin \phi=\sqrt{3 /\left(4+k^{2}\right)},
$$

where $k$ is related to the perpendicular height of the constituent pyramids and is given by the positive root of

$$
\lambda k^{2}+\mu k+v=0 .
$$

Here the coefficients $\lambda, \mu$ and $v$ are given by equations:

$$
\begin{gathered}
\lambda=1-\sin ^{2} \omega \cos ^{2} \theta, \\
\mu=2 \cos \omega \cos \theta(\sin \omega \cos \theta+\sqrt{3} \cot \psi), \\
v=1-\cos ^{2} \theta\left(4-\sin ^{2} \omega\right)-2 \sqrt{3} \sin \omega \cos \theta \cot \psi,
\end{gathered}
$$

which in turn lead to

$$
k=\frac{-\mu+\sqrt{\mu^{2}-4 \lambda v}}{2 \lambda}
$$

Substituting for $k$ yields the equation, from which the angle $\phi$ can be determined. The task of determining all the angles for the problem is now complete and therefore 
the diameter can be calculated:

$$
\frac{D_{(n, m)}}{d_{(n, m)}}=\frac{2 \sin \phi \cos \theta}{\sin \psi} .
$$

Summarizing above description, it should be stated that calculation of the nanotubes' diameter in the frames of idealized polyhedral model seems to be a tedious numerical procedure.

\subsection{Analytical Polyhedral Model}

In general, to describe a graphitic nanotube as a polyhedron one must begin with the tessellation of regular hexagons where the vertices of the tessellation represent the atoms and lines of the hexagons represent chemical bonds.

In their model, Cox and Hill overlay on this a second tessellation of equilateral triangles where the vertices of the triangles are the atoms and every second triangle also has an atom located at its center. The net effect of these two tessellations is a single tessellation of equilateral and isosceles triangles and by fixing the lengths of the sides, which represent bonds, it is possible to construct a truly facetted polyhedron where all vertices are equidistant from an axis of symmetry and all the bond lengths and bond angles are equal for all atoms.

At the same time, one of us-Chkhartishvili-suggested another version of polyhedral model for achiral, i.e., zigzag and armchair, boron nitride nanotubes with equal bond lengths and also rolled up from a hexagonal plane sheet, in which however sites were alternatively occupied by boron and nitrogen atoms [29-32]. Our model uses different method of tessellation: equilateral hexagons in zigzag and armchair sheets are divided into two isosceles trapeziums or one rectangle and two isosceles triangles, respectively. It means that all of lines of tessellation are parallel to the tube axis.

Nanotubes' cylindrical symmetry accounted in this way allows us obtaining of the explicit expressions for atomic sites co-ordinates, inter-atomic distances, and diameters of zigzag

$$
\frac{D_{(n, 0)}}{d_{(n, 0)}}=\frac{\sqrt{3}}{2 \sin \pi / 2 n}
$$

and armchair

$$
\frac{D_{(n, n)}}{d_{(n, n)}}=\frac{\sqrt{5+4 \cos \pi / 2 n}}{2 \sin \pi / 2 n}
$$

nanotubes. From these expressions it is easy to construct an interpolation formula

$$
\frac{D_{(n, m)}}{d_{(n, m)}} \approx \frac{\sqrt{3+(2 m / n)(1+\cos \pi / 2 n)}}{2 \sin \pi / 2 n}
$$

useful for chiral nanotubes as well.

Thus, in this approach nanotubes' diameter is calculated analytically. By reason of this, let call our model as analytical polyhedral model.

In general, only geometrical consideration will be insufficient: the equilibrium bond length value in nanotube should be found maximizing its molar binding energy. Description made for the nanotubular geometry may serve as basis for further ground state and electron structure calculations. In particular, quasi-classical approach can be used for this purpose (physical theory and related mathematical problems see in [33] and [34,35], respectively), which successfully have been applied to other structural modifications of boron nitride. Recently, the relative stability of the small- and intermediate-diameter $\mathrm{BN}$ nanotubes has been studied by this method, and a complex dependence of the molar energy on the diameter was obtained $[30,36]$. As for the equilibrium bonds length, for all calculated single-walled boron nitride nanotubes it is found to be in satisfactory agreement with experimental value obtained for the intra-layer bonds length in BN layered crystals. This fact enables us to use experimental bond length values in all graphitic-type crystals in estimates made for corresponding nanotubular structures.

Let draw an analogy with other theoretical (namely, Monte Carlo) simulations performed in [37-40] to obtain the dependence of morphology and configuration of the assembled polymer cylindrical nanostructures confined in nanopores on their diameters and surface conditions.

\section{Recent Experimental Data and Their Analysis}

An ideal experimental characterization method of nanotube structures would require little sample preparation, minimize potential damage to single-wall nanostructures, and allow the characterized tubes to be used for subsequent experiments or device fabrication. Optical imaging of carbon nanotubes placed directly on a solid substrate meets these requirements and allows rapid visualization and spectral resolution of individual nanotubes with relative ease. Recently, it has been reported [41] a novel onchip Rayleigh imaging technique using wide-field laser illumination to measure optical scattering from individual single-walled carbon nanotubes with high spatial and spectral resolution. This method in conjunction with calibrated atomic force microscopy accurately measures the resonance energies and diameters for a large number of tubes in parallel.

The technique was applied for fast mapping of key parameters, including the chiral indices for individual single-walled carbon nanotubes. The values of diameters 
and chiral indices experimentally determined in [41] are shown in Table 1.

In the present work, we have calculated same nanotubular diameters based on the analytical polyhedral model's interpolation formula for given chiral indices and for following bond lengths values $0.140,0.141,0.142,0.143$ and $0.144 \mathrm{~nm}$ from the vicinity of $0.142 \mathrm{~nm}$, the bond length value in the graphene (see for example [2]). The best agreement with measured and calculated values was found just for the bond length of $0.142 \mathrm{~nm}$ characteristic for carbon honeycomb plane sheet. These theoretical results also are listed in Table $\mathbf{1 .}$

One can see that for three, two, two and three species relative difference between experiment and theory are almost $0,<1,<3$, and $<12 \%$, respectively. Agreement can be improved - made all deviations less than 1\%slightly (not more than in \pm 2 ) changing chiral indices. Refined chiral indices, diameters and corresponding lowered deviations are shown in brackets.

The possibility of refinement in chiral indices reveals the fact experimentally obtained in [41] that frequent chirality-changing structural defects accompanied with only slight diameter-changes are characteristic for grown single-walled carbon nanotubes. Consequently, it is not improbable that measured values of diameter, on the one hand, and chiral indices, on the other hand, are attributed with different parts of the same nanotube.

In general, electronic and other significant properties of the single-walled nanotubes depend on their structure, which may be characterized by two parameters: the diameter and the chirality, which can be encoded by two integers - nanotube indices $(n, m)$. Usually, for the synthesis of carbon nanotubes one may achieve some control over their diameters but little control over their chiralities. As such tubes may be either metallic or semi- conducting this poor structural control implies a rather poor control over their electronic properties. And for nanotubular systems other than carbon, one faces almost the same situation. The basic reason for this defect has been clearly explained in [42].

According to this explanation, the noted fact that the chiral indices vary for an almost fixed tube diameter is a direct consequence of the carbon nanotube strain energy dependence upon its diameter. By virtue of this dependence, the synthesis conditions fix a certain range of diameters but leave the chirality practically unspecified. Strain energy quantifies 1) the difference in cohesive energy among different nanotubes, 2) the deformation (curvature) energy per atom which is necessary to roll up a single sheet into a nanotube of certain diameter and chirality, and 3) a measure of the mechanical tension of a nanotube - this tension stabilizes the tubular shape and makes the tube round.

In particular, for carbon nanotubes the strain energy refers to a graphene sheet and the strain energy effectively depends on the diameter but not on the chirality. The radial dependence is easy to understand: the diameter is just a measure for the curvature of a tube, and the smaller its diameter the more energy is needed to bend a graphene sheet. But the independence of strain energy of chirality may be attributed to the nearly isotropic inplane mechanical properties of the graphene sheet, as quantified by its elastic moduli for stretching and bending. For example, the diagonal elements of the elastic tensor of graphene are the same, due to a hexagonal symmetry of the honeycomb lattice. Therefore, when stretching a graphene sheet along different in-plane lattice directions, one will observe the same stiffness, and the physical systems will behave like a homogeneous two-dimensional continuum. Thus, when rolling up a

Table 1. Experimental and theoretical values of single-walled carbon nanotubes' diameters.

\begin{tabular}{|c|c|c|c|c|c|c|c|}
\hline \multirow{2}{*}{$\begin{array}{l}\# \\
1\end{array}$} & \multicolumn{2}{|c|}{$(n, m)$} & \multicolumn{2}{|c|}{$D_{(n, m)}^{\text {Theo }}, \mathrm{nm}$} & \multirow{2}{*}{$\frac{D_{(n, m)}^{E x p}, \mathrm{~nm}}{1.58}$} & \multicolumn{2}{|c|}{$|\delta|, \%$} \\
\hline & $(12,11)$ & {$[(14,11)]$} & 1.40 & {$[1.57]$} & & 11.4 & [0.6] \\
\hline 2 & $(20,4)$ & & 1.76 & & 1.77 & 0.0 & \\
\hline 3 & $(22,2)$ & & 1.83 & & 1.83 & 0.0 & \\
\hline 4 & $(15,14)$ & {$[(17,16)]$} & 1.76 & [2.00] & 1.99 & 11.6 & [0.5] \\
\hline 5 & $(20,9)$ & {$[(22,7)]$} & 1.98 & [2.06] & 2.04 & 2.9 & [1.0] \\
\hline 6 & $(16,15)$ & {$[(18,17)]$} & 1.88 & [2.12] & 2.13 & 11.7 & [0.5] \\
\hline 7 & $(13,1)$ & & 1.07 & & 1.07 & 0.0 & \\
\hline 8 & $(15,0)$ & & 1.18 & & 1.19 & 0.8 & \\
\hline 9 & $(23,5)$ & & 2.05 & & 2.05 & 0.0 & \\
\hline 10 & $(25,10)$ & {$[(25,11)]$} & 2.42 & [2.47] & 2.48 & 2.4 & [0.4] \\
\hline
\end{tabular}


graphene sheet along different in-plane directions to form various nanotubes with similar diameters, this process will require almost same deformation energy.

A similar behavior is also known for boron nitride $\mathrm{BN}$ and other binary compounds nanotubes graphitic structure. It was shown [43] that main point defects formed in the BN single-walled nanotubes are vacancies, which tend to associate in divacancies. Due to the partially ionic character of the chemical bonding in $\mathrm{BN}$, divacancies behave like a Schottky pair, with very high dissociation energy. Clustering of multiple vacancies in boron nitride nanotubes being energetically favorable leads to extended defects formation, which locally change the nanotube diameter and chirality. Nevertheless, these defects do not alter significantly the band gap energy and electronic structure in whole, from which are originated optical properties of nanotubes used in structural studies.

Finally, it should be mentioned that asymptotical analysis $(n>>1)$ of the nanotube diameter formula according to our model, as well as numerical estimates made based on same formula for boron nitride reveal number of groups of nanotubes with almost same diameters, but different chiralities [32].

\section{Conclusions}

Summarizing obtained results, we can conclude that due to the isotropic in-plane mechanical properties of the honeycomb plane sheet, the energy needed to roll up a nanotube is almost independent of the roll up direction, i.e. chiral indices. It leads to the chiralty-changing structural defects and as a result to experimental ambiguities in determination of chiral indices. These ambiguities can be avoided characterizing nanotube structure by the analytical polyhedral model.

\section{References}

[1] S. Iijima, "Helical Microtubules of Graphitic Carbon," Nature, Vol. 354, No. 6348, 1991, pp. 56-58. doi: $10.1038 / 354056 \mathrm{a} 0$

[2] A. Jorio, M. S. Dresselhaus, M. Saito and G. Dresselhaus, "Raman Spectroscopy in Graphene Related Systems," Wiley-VCH, Berlin, 2011. doi:10.1002/9783527632695

[3] N. G. Chopra, R. J. Luyken, K. Cherrey, V. H. Crespi, M. L. Cohen, S. G. Louie and A. Zettl, "Boron Nitride Nanotubes," Science, Vol. 269, No. 5226, 1995, pp. 966967. doi:10.1126/science.269.5226.966

[4] Y. Zhang, K. Suenaga, C. Colliex and S. Iijima, "Coaxial Nanocable: Silicon Carbide and Silicon Oxide Sheathed with Boron Nitride and Carbon," Science, Vol. 281, No. 5379, 1998, pp. 973-975. doi:10.1126/science.281.5379.973

[5] X. Blasé, J.-C. Charlier, A. de Vita and R. Car, "Structur- al and Electronic Properties of Composite $\mathrm{B}_{x} \mathrm{C}_{y} \mathrm{~N}_{z}$ Nanotubes and Heterojunctions," Applied Physics A, Vol. 68, No. 3, 1999, pp. 293-300. doi:10.1007/s003390050891

[6] V. V. Pokropivny, V. V. Skorokhod, G. S. Oleinik, A. V. Kurdyumov, T .S. Bartnitskaya, A. V. Pokropivny, A. G. Sisonyuk and D. M. Sheichenko, "Boron Nitride Analogs of Fullerenes (the Fulborenes), Nanotubes, and Fullerites (the Fulborenites)," Journal of Solid State Chemistry, Vol. 154 , No. 1, 2000, pp. 214-222. doi: $10.1006 /$ jssc. 2000.8838

[7] S. M. Nakhmanson, A. Cazolari, V. Meunier, J. Bernholc and M. B. Nardeli, "Spontaneous Polarization and Piezoelectricity in Boron Nitride Nanotubes," Physical Review B, Vol. 67, No. 23, 2003, 5 Pages.

[8] N. Sai and E. J. Mele, "Microscopic Theory for Nanotube Piezoelectricity," Physical Review B, Vol. 68, No. 24, 2003, 3 Pages.

[9] W.-Q. Han, C. W. Chang and A. Zettl, "Encapsulation of One-Dimensional Potassium Halide Crystals within BN Nanotubes," Nano Letters, Vol. 4, No. 7, 2004, pp. 14551357. doi: $10.1021 / \mathrm{nl} 0494452$

[10] K. Yum and M.-F. Yu, "Measurement of Wetting Properties of Individual Boron Nitride Nanotubes with the Wilhelmy Method Using a Nanotube-Based Force Sensor," Nano Letters, Vol. 6, No. 2, 2006, pp. 329-333. doi:10.1021/n10520841

[11] C. Zhi, Y. Bando, C. Tang and D. Golberg, "Engineering of Electronic Structure of Boron-Nitride Nanotubes by Covalent Functionalization," Physical Review B, Vol. 74, No. 15, 2006, 4 Pages.

[12] S. A. Shevlin and Z. X. Guo, "Hydrogen Sorption in Defective Hexagonal BN Sheets and BN Nanotubes," Physical Review B, Vol. 76, No. 2, 2007, 11 Pages.

[13] E. Durgun, Y.-R. Jang and S. Ciraci, "Hydrogen Storage Capacity of Ti-doped Boron-Nitride and B/Be-Substituted Carbon Nanotubes," Physical Review B, Vol. 76, No. 7, 2007, 4 Pages.

[14] Q. Wu, Z. Hu, X. Wang, Y. Lu, X. Chen, H. Xu and Y. Chen, "Synthesis and Characterization of Faceted Hexagonal Aluminum Nitride Nanotubes," Journal of the American Chemical Society, Vol. 125, No. 34, 2003, pp. 10176-10177. doi:10.1021/ja0359963

[15] J. Goldberger, R. He, Y. Zhang, S. Lee, H. Yan, H.-J. Choi and P. Yang, "Single-Crystal Gallium Nitride Nanotubes," Nature, Vol. 422, No. 6932, 2003, pp. 599-602. doi:10.1038/nature01551

[16] J. Q. Hu, Q. Li, X. M. Meng, C. S. Lee and S. T. Lee, "Thermal Reduction Route to the Fabrication of Coaxial $\mathrm{Zn} / \mathrm{ZnO}$ Nanocables and ZnO Nanotubes," Chemistry of Materials, Vol. 15, No. 1, 2003, pp. 305-308. doi: $10.1021 / \mathrm{cm} 020649 \mathrm{y}$

[17] Y. J. Xing, Z. H. Xi, Z. Q. Xue, X. D. Zhang, J. H. Song, R. M. Wang, J. Xu, Y. Song, S. L. Zhang and D. P. Yu, "Optical Properties of the ZnO Nanotubes Synthesized via Vapor Phase Growth," Applied Physics Letters, Vol. 83, No. 9, 2003, pp. 1689-1691. doi:10.1063/1.1605808 
[18] X.-H. Zhang, S.-Y. Xie, Zh.-Y. Jiang, X. Zhang, Z.-Q. Tian, Z.-X. Xie, R.-B. Huang and L.-S. Zheng, "Rational Design and Fabrication of ZnO Nanotubes from Nanowire Templates in a Microwave Plasma System," Journal of Physical Chemistry B, Vol. 107, No. 37, 2003, pp. 10114-10118. doi:10.1021/jp034487k

[19] S. Erkoc and H. Kökten, "Structural and Electronic Properties of Single-Wall ZnO Nanotubes," Physica E, Vol. 28, No. 2, 2005, pp. 162-170. doi:10.1016/j.physe.2005.03.004

[20] H. Zhang, S. Zhang, S. Pan, G. Li and J. Hou, "A Simple Solution Route to ZnS Nanotubes and Hollow Nanospheres and Their Optical Properties," Nanotechnology, Vol. 15, No. 8, 2004, pp. 945-948. doi:10.1088/0957-4484/15/8/012

[21] Y.-C. Zhu, Y. Bando and Y. Uemura, "ZnS-Zn Nanocables and ZnS Nanotubes," Chemical Communications, No. 7, 2003, pp. 836-837. doi:10.1039/b300249g

[22] M. S. Dresselhaus, G. Dresselhaus and R. Saito, "Carbon Fibers Based on $\mathrm{C}_{60}$ and Their Symmetry," Physical Review B, Vol. 45, No. 11, 1992, pp. 6234-6242. doi:10.1103/PhysRevB.45.6234

[23] R. A. Jishi, M. S. Dresselhaus and G. Dresselhaus, "Symmetry Properties of Chiral Carbon Nanotubes," Physical Review B, Vol. 47, No. 24, 1993, pp. 1667116674. doi:10.1103/PhysRevB.47.16671

[24] M. S. Dresselhaus, G. Dresselhaus and R. Saito, "Physics of Carbon Nanotubes," Carbon, Vol. 33, No. 7, 1995, pp. 883-891.

[25] A. Rubio, J. L. Corkill and M. L. Cohen, "Theory of Graphitic Boron Nitride Nanotubes," Physical Review B, Vol. 49, No. 7, 1994, pp. 5081-5084.

[26] B. J. Cox and J. M. Hill, "Exact and Approximate Geometric Parameters for Carbon Nanotubes Incorporating Curvature," Carbon, Vol. 45, No. 7, 2007, pp. 14531462. doi:10.1016/j.carbon.2007.03.028

[27] B. J. Cox and J. M. Hill, "Geometric structure of U1tra-Small Carbon Nanotubes," Carbon, Vol. 46, No. 4, 2008, pp. 711-713. doi:10.1016/j.carbon.2007.12.011

[28] R. K. F. Lee, B. J. Cox and J. M. Hill, "The Geometric Structure of Single-Walled Nanotubes," Nanoscale, Vol. 2, No. 6, 2010, pp. 859-872. doi:10.1039/b9nr00433e

[29] L. S. Chkhartishvili, "On Sizes of Boron Nitride Nanotubes," In: I. M. Neklyudov and V. M. Shulayev, Eds., Thin Films in Optics and Nanoelectronics, NSC "KhIPT"-PPP “Contrast", Kharkiv, 2006, pp. 367-373.

[30] L. S. Chkhartishvili, "Equilibrium Geometry of UltraSmall-Radius Boron Nitride Nanotubes," Material Science of Nanostructures, No. 1, 2009, pp. 33-44.

[31] L. Chkhartishvili, "Boron Nitride Nanosystems of Regu- lar Geometry," Journal of Physics: Conference Series, Vol. 176, No. 1, 2009, 17 Pages.

[32] L. S. Chkhartishvili and T. M. Berberashvili, "Sequences of Layers in Binary Compounds Multi-Walled Nanotubes and Multi-Shelled Fullerenes," Material Science of $\mathrm{Na}$ nostructures, No. 3, 2010, pp. 20-28.

[33] L. Chkhartishvili, "Quasi-Classical Theory of Substance Ground State," Technical University Press, Tbilisi, 2004.

[34] L. S. Chkhartishvili, "Volume of the Intersection of Three Spheres," Mathematical Notes, Vol. 69, No. 3, 2001, pp. 421-428. doi:10.1023/A:1010295711303

[35] L. S. Chkhartishvili, "Iterative Solution of the Secular Equation," Mathematical Notes, Vol. 77, No. 1, 2005, pp. 273-279. doi:10.1007/s11006-005-0026-y

[36] L. Chkhartishvili and I. Murusidze, "Molar Binding Energy of Zigzag and Armchair Single-Walled Boron Nitride Nanotubes," Materials Sciences and Applications, Vol. 1, No. 4, 2010, pp. 223-246. doi:10.4236/msa.2010.14035

[37] H. Chen and E. Ruckenstein, "The Driving Force of Channel Formation in Triheteropolymers Confined in Nanocylindrical Tubes," Journal of Chemical Physics, Vol. 130, No. 2, 2009, 5 Pages.

[38] H. Chen and E. Ruckenstein, "Nanostructures Self-Assembled in Polymer Solutions Confined in Cylindrical Nanopores," Langmuir, Vol. 25, No. 20, 2009, pp. 12315-12319. doi:10.1021/la901571m

[39] H. Chen and E. Ruckenstein, "The Structure of Nanochannels Formed by Block Copolymer Solutions Confined in Nanotubes," Journal of Chemical Physics, Vol. 131, No. 11, 2009, 6 Pages.

[40] H. Chen and E. Ruckenstein, "Relation between Molecular Orientation and Morphology of a Multiblock Copolymer Melt Confined in Cylindrical Nanopores," Polymer, Vol. 51, No. 4, 2010, pp. 968-974.

doi:10.1016/j.polymer.2010.01.002

[41] D. Y. Joh, L. H. Herman, S.-Y. Ju, J. Kinder, M. A. Segal, J. N. Johnson, G. K. L. Chan and J. Park, "On-Chip Rayleigh Imaging and Spectroscopy of Carbon Nanotubes," Nano Letters, Vol. 11, No. 1, 2011, pp. 1-7. doi: $10.1021 / \mathrm{nl} 1012568$

[42] J. Kunstman, A. Quandt and I. Boustani, "An Approach to Control the Radius and the Chirality of Nanotubes," Nanotechnology, Vol. 18, No. 15, 2007, 3 Pages.

[43] A. Zobelli, C. P. Ewels, A. Gloter, G. Seifert, O. Stephan, S. Csillag and C. Colliex, "Defective Structure of BN Nanotubes: From Single Vacancies to Dislocation Lines," Nano Letters, Vol. 6, No. 9, 2006, pp. 1955-1960. doi: $10.1021 / \mathrm{n} 10610811$ 